\author{
Military Technical College \\ Kobry El-Kobba \\ Cairo, Egypt
}

12-th International Conference on

Aerospace Sciences \& Aviation Technology

\title{
ACTIVE CONTROL OF INTERIOR NOISE IN A RECTANGULAR CAVITY WITH TWO FLEXIBLE PANELS
}

\author{
MOHAMED* GHARIB ${ }^{1}$ and MOHAMMAD ${ }^{* *}$ TAWFIK $^{*}$
}

\begin{abstract}
In this work, a numerical effort is presented for modelling and control of structureacoustics coupled systems. Modelling of sound transmission through a panel-cavitypanel system is presented. An approximate series solution is assumed and the solution is obtained using Galarkin's method. The system to be modelled is consisting of a rectangular cavity with two flexible panels, one at the top of the cavity while the other at the bottom and four other fixed boundaries. PZT pair patches are considered to be bonded to the top panel, and each pair is assumed to produce a pure moment actuation when an electric drive signal is used to excite them. The flexible panel is exposed to an external pressure excitation due to a planar wave generated by a sound source mounted above the cavity. Displacements at the mid points are calculated for the upper and lower panels. The developed model is controlled using the optimal LQR control law. The numerically obtained time responses from the compensated model are found to be acceptable compared to the uncompensated ones. It is found that the actuation of the upper panel can decrease the vibration of the lower one rather than decreasing the acoustic pressure inside the cavity.
\end{abstract}

\section{KEY WORD:}

Active Control, Structural-Acoustic interaction and Piezoelectric Materials

\footnotetext{
* Graduate Student, Vibration and Acoustics Control Center, Noon for Researches and Developments, www.noonrd.org, Cairo, Egypt.

${ }^{* *}$ Assistant Professor, Modelling \& Simulation in Mechanics Department, Faculty of Engineering \& Material Science, German University in Cairo, New Cairo City, Egypt.
} 


\section{INTRODUCTION}

The purpose of this work is to model and control the structural-acoustic system. The system to be modelled is consisting of a rectangular cavity with two flexible panels, one at the top and the other at the bottom and four other fixed boundaries. PZT pair patches are considered to be bonded to the top panel (Fig.1), and each pair is assumed to produce a pure moment actuation when an electric drive signal is used to excite them. The flexible panel is exposed to an external pressure excitation due to a planar wave generated by a sound source mounted above the cavity. The inner dimensions of the cavity are $L_{x c}, L_{y c}$ and $L_{z c}$, in the $x, y$, and $z$ directions respectively, and the effective dimensions of the panel are $L_{x p}$ and $L_{y p}$. As shown in the schematic of the panel-cavity-panel system (Figure 1), two coordinate systems are used to describe the system; the first one with the origin at $O_{c}$ is used for the cavity, and the second one with the origin at $O_{p}$ is used for the panels. The panel may have larger dimensions than the cavity. The two plates are set such that one of them is at the top of the cavity at $z=L_{z c}$, while the other is at the bottom of the cavity at $z=0$. Throughout the analysis, the ambient values are indicated with the subscript ( $)_{0}$. For convenience, the structure-acoustic system modelling is divided into the following subsystems: (i) The plate-cavity-plate system, (ii) The plate-piezo system, and (iii) The piezo-plate-cavity-plate system.

\section{MODELLING OF THE PANEL-CAVITY-PANEL PROBLEM}

\subsection{The panel-cavity-panel system}

The two governing equations of this system are the conservation of mass equation and the conservation of momentum equation. In three-dimensional space, making use of linear approximations, the wave equation describing the sound field inside the cavity can be obtained as:

$$
\nabla^{2} P-\frac{1}{c_{o}^{2}} \frac{\partial^{2} P}{\partial t^{2}}=0
$$

where $P(x, y, z ; t)$ is the air pressure inside the cavity, the speed of sound in a medium assuming isentropic flow is defined as [1]

$$
c=\sqrt{\frac{d P}{d \rho}}
$$

At a rigid boundary, the normal component of the air particle velocity is set to zero, and at a flexible boundary, it is set equal to the normal velocity of the flexible panel. Thus, the boundary conditions can be stated as:

$$
\frac{\partial P}{\partial n}=\left\{\begin{array}{cl}
0 & \text { at rigid boundary } \\
-\rho_{o} \frac{\partial^{2} w}{\partial t^{2}} & \text { at flexible boundary }
\end{array}\right.
$$


where $w(x, y ; t)$ is the normal displacement of the flexible boundary, and $n$ is the direction normal to the boundary. The pressure field inside the cavity can be expressed in the series form

$$
P(x, y, z ; t)=\sum_{i=1}^{\infty} \Phi_{i}(x, y, z) q_{i}(t)=\sum_{i=1}^{\infty} \psi_{i}(x) \varphi_{i}(y) \Gamma_{i}(z) q_{i}(t)
$$

where $\Phi_{i}(x, y, z)$ are used to describe the spatial field and $q_{i}(t)$ are used to describe the associated temporal part of the pressure response. The spatial functions $\psi_{i}(x), \varphi_{i}(y)$ and $\Gamma_{i}(z)$ are assumed to be orthogonal. Substituting Eq. (4) into Eq. (1), integrating over the volume of the cavity (Galarkin's approach), and making use of the orthogonality conditions and the boundary conditions, the cavity governing equations can be derived to have the following form

$$
\frac{1}{c_{o}^{2}} \frac{\partial^{2} q_{i}}{\partial t^{2}}+\left[k_{i}^{2}-\left.\left(\bar{\Gamma}_{i} \frac{d \bar{\Gamma}_{i}}{d z}\right)\right|_{o_{c}} ^{L_{c c}}\right] q_{i}=0
$$

where

$$
k_{i}^{2}=\left[\int_{o_{c}}^{L_{x c}}\left(\frac{d \psi_{i}}{d x}\right)^{2} d x+\int_{o_{c}}^{L_{y c}}\left(\frac{d \varphi_{i}}{d y}\right)^{2} d y+\int_{o_{c}}^{L_{z c}}\left(\frac{d \Gamma_{i}}{d z}\right)^{2} d z\right]
$$

Eq. (5) will be used later with the panel-piezo equations to satisfy the boundary condition at the flexible panel.

\subsection{The piezoelectric actuator-panel system}

The panel-piezo system is treated here as a multi-laminate system that consists of three plies in places where the piezo pair patches are bonded to the panel, and as a single ply panel otherwise. Making use of the assumptions used in earlier studies [3], the panel displacement can be described by

$$
D \nabla^{4} w+\rho_{p} h_{p} \ddot{w}=p_{i n}-p_{i}-\sum_{i=1}^{k} \frac{\left(h_{p}-h_{p z t}\right) E_{p z t} d_{31}}{(1-v)} \nabla^{2} \chi_{i} V_{i}(t)
$$

The plate response is assumed in the series

$$
w(x, y ; t)=\sum_{i=1}^{\infty} \alpha_{i}(x) \beta_{i}(y) \eta_{i}(t)
$$

where the $\eta_{i}(t)$ are temporal functions and the appropriate expressions for the spatial functions $\alpha_{i}(x)$ and $\beta_{i}(y)$ are obtained from the work of [5]. The upper plate only has the PZT patches and the incident pressure waves are excite it, then the equations governing the two plates can be written as 


$$
\begin{gathered}
D \nabla^{4} w_{U}+\rho_{p} h_{p} \ddot{w}_{U}=p_{i n}-p_{U i}-\sum_{i=1}^{k} \frac{\left(h_{p}-h_{p z t}\right) E_{p z t} d_{31}}{(1-v)} \nabla^{2} \chi_{U i} V_{i}(t) \\
D \nabla^{4} w_{L}+\rho_{p} h_{p} \ddot{w}_{L}=p_{\text {in }}
\end{gathered}
$$

where the subscript ( $)_{U}$ and ( $)_{L}$ are used to describe the upper and lower panels respectively.

\subsection{The coupled piezo-panel-cavity-panel system}

In this section, the panel-cavity system is coupled with the piezoelectric actuatorpanel system to obtain the governing equations for the coupled cavity subsystem. The boundary conditions at the flexible boundary are recalled from Eqs. (3)

$$
\begin{aligned}
& \left.\frac{\partial P}{\partial z}\right|_{z=o_{c}}=-\rho_{o} \frac{\partial^{2} w_{L}}{\partial t^{2}} \\
& \left.\frac{\partial P}{\partial z}\right|_{z=L_{z c}}=-\rho_{o} \frac{\partial^{2} w_{U}}{\partial t^{2}}
\end{aligned}
$$

Making use of this boundary condition along with Eqs. (4) and (8), and making use of the orthogonality property, we get the following equation:

$$
\begin{aligned}
& \left.\frac{\partial \Gamma_{j}}{\partial z}\right|_{z=L_{z c}} q_{j}(t)=-\rho_{o} \sum_{i=1}^{\infty} B x_{i j}^{(c)} B y_{i j}^{(c)} \ddot{\eta}_{U}(t) \\
& \left.\frac{\partial \Gamma_{j}}{\partial z}\right|_{z=0_{o}} q_{j}(t)=-\rho_{o} \sum_{i=1}^{\infty} B x_{i j}^{(c)} B y_{i j}^{(c)} \ddot{\eta}_{L}(t)
\end{aligned}
$$

where

$$
B x_{i j}^{(c)}=\int_{o_{c}}^{L_{x c}} \alpha_{i}(x) \psi_{i}(x) d x \quad B y_{i j}^{(c)}=\int_{o_{c}}^{L_{x c}} \beta_{i}(y) \varphi_{i}(y) d y
$$

After substituting Eq. (11) into Eqs. (5) and (6), we get the equation governing a pressure-field mode as follows:

$$
\frac{1}{c_{o}{ }^{2}} \ddot{q}_{j}(t)+k_{j}{ }^{2} q_{j}(t)-\left(-\rho_{o} \sum_{i=1}^{\infty} B x_{i j}^{(c)} B y_{i j}^{(c)}\right)\left(\left.\Gamma_{j}\right|_{z=L_{z c}} \ddot{\eta}_{U}(t)-\left.\Gamma_{j}\right|_{z=L_{z c}} \ddot{\eta}_{L}(t)\right)=0
$$

The last terms on the left-hand side of Eq. (13) represent the structural-acoustic coupling in the system. At this stage, it is assumed that the spatial functions in Eq. (4) are given by rigid-body cavity modes; that is [2],[3], and [4] 


$$
\psi_{i}(x)=\frac{A_{i}}{\sqrt{L_{x c}}} \cos \left(\frac{l_{i} \pi x}{L_{x c}}\right), \quad \varphi_{i}(y)=\frac{A_{i}}{\sqrt{L_{y c}}} \cos \left(\frac{m_{i} \pi y}{L_{y c}}\right), \quad \Gamma_{i}(z)=\frac{A_{i}}{\sqrt{L_{z c}}} \cos \left(\frac{n_{i} \pi z}{L_{z c}}\right)
$$

where the indices $l_{i}, m_{i}$, and $n_{i}$ are associated with the spatial functions of the $i^{\text {th }}$ rigid cavity mode, in the $x, y$, and $z$ directions, respectively. The constants $A_{i}$ are chosen to satisfy the orthogonality conditions. Using Eq. (14) the spatial function $\Gamma_{j}(z)$ at each plate will be

$$
\left.\left(\Gamma_{j}(z)\right)\right|_{\text {at plate }}=\left\{\begin{array}{l}
\left(\Gamma_{j}(z)\right)_{z=L z c}=\frac{(-1)^{j} A_{j}}{\sqrt{L_{z c}}} \text { at upper plate } \\
\left(\Gamma_{j}(z)\right)_{z=0}=\frac{A_{j}}{\sqrt{L_{z c}}} \text { at lower plate }
\end{array}\right.
$$

Making use of Eq. (15) in Eq. (13), it is found that

$$
\begin{aligned}
\left(\frac{1}{c_{o}^{2}}\right) \ddot{q}_{j}(t) & +\left(\frac{l_{j}^{2} \pi^{2}}{L_{x c}^{2}}+\frac{m_{j}^{2} \pi^{2}}{L_{z c}^{2}}+\frac{n_{j}^{2} \pi^{2}}{L_{z c}^{2}}\right) q(t) \\
& +\left(\frac{(-1)^{j} A_{j}}{\sqrt{L_{z c}}} \rho_{o} \sum_{i=1}^{\infty} B x_{i j}^{(c)} B y_{i j}^{(c)}\right) \ddot{\eta}_{U}(t)-\left(\frac{A_{j}}{\sqrt{L_{z c}}} \rho_{o} \sum_{i=1}^{\infty} B x_{i j}^{(c)} B y_{i j}^{(c)}\right) \ddot{\eta}_{L}(t)=0
\end{aligned}
$$

The equations governing the panel modal amplitudes are obtained by making use of Eqs. (4), (7), (8) and (14). After making use of the orthogonality properties and boundary conditions, the equation governing each panel modal amplitude is obtained as:

$$
\begin{gathered}
\rho_{p} h_{p} \ddot{\eta}_{U j}(t)+D\left[I x_{j}+I y_{j}\right] \eta_{U j}(t)+2 D \sum_{i=1}^{\infty} I x_{i j} I y_{i j} \eta_{U i}(t) \\
=\sum_{i=1}^{\infty} \frac{(-1)^{j} A_{i}}{\sqrt{L_{z c}}} B x_{i j}^{(p)} B y_{i j}^{(p)} q_{i}(t)-\left[\int_{A_{p}} \alpha_{j} \beta_{j} p_{U i}^{s}(x, y) \mathrm{d} A_{p}\right] p_{i}^{t}(t) \\
-\sum_{i=1}^{k}\left[\int_{A_{p}} \alpha_{j} \beta_{j} \frac{\left(h_{p}+h_{p t t}\right) E_{p z t} d_{31}}{(1-v)} \nabla^{2} \chi_{U}\left(x_{i}, y_{i}\right) \mathrm{d} A_{p}\right] V_{i}(t) \\
\rho_{p} h_{p} \ddot{\eta}_{L j}(t)+D\left[I x_{j}+I y_{j}\right] \eta_{L j}(t)+2 D \sum_{i=1}^{\infty} I x_{i j} I y_{i j} \eta_{L i}(t) \\
=\sum_{i=1}^{\infty} \frac{A_{i}}{\sqrt{L_{z c}}} B x_{i j}^{(p)} B y_{i j}^{(p)} q_{i}(t)
\end{gathered}
$$

where the different spatial integrals are given by 


$$
\begin{array}{ll}
B x_{i j}^{(p)}=\int_{o_{c}}^{L_{x c}} \psi_{i}(x) \alpha_{j}(x) d x & B y_{i j}^{(p)}=\int_{o_{c}}^{L_{y c}} \varphi_{i}(y) \beta_{j}(y) d y \\
I x_{j}=\int_{o_{c}}^{L_{x c}} \alpha_{j}(x) \frac{d^{4} \alpha_{j}(x)}{d x^{4}} d x & I y_{j}=\int_{o_{c}}^{L_{y c}} \beta_{j}(y) \frac{d^{4} \beta_{j}(y)}{d y^{4}} d y \\
I x_{i j}=\int_{o_{c}}^{L_{x c}} \frac{d^{2} \alpha_{i}(x)}{d x^{2}} \alpha_{j}(x) d x & I y_{i j}=\int_{o_{c}}^{L_{y c}} \frac{d^{2} \beta_{i}(y)}{d y^{2}} \beta_{j}(y) d y
\end{array}
$$

In Eq. (17), the incident pressure loading can be expressed as the product of spatial and time domain functions; that is,

$$
p_{i}(x, y ; t)=p_{i}^{s}(x, y) p_{i}^{t}(t)
$$

Now, Eqs. (16) and (17) can be represented in matrix from, after truncating the infinite number of modes to the first $M$ panel modes and $N$ acoustic modes, as follows:

$$
\left[\begin{array}{ccc}
M_{p p}^{U} & 0 & 0 \\
M_{c p}^{U} & M_{c c} & M_{c p}^{L} \\
0 & 0 & M_{p p}^{L}
\end{array}\right]\left(\begin{array}{c}
\ddot{\eta}_{U} \\
\ddot{q} \\
\ddot{\eta}_{L}
\end{array}\right]+\left[\begin{array}{ccc}
K_{p p}^{U} & K_{p c}^{U} & 0 \\
0 & K_{c c} & 0 \\
0 & K_{p c}^{L} & K_{p p}^{L}
\end{array}\right]\left(\begin{array}{c}
\eta_{U} \\
q \\
\eta_{L}
\end{array}\right)=\left[\begin{array}{cc}
F_{p}^{U} & F_{V}^{U} \\
0 & 0 \\
0 & 0
\end{array}\right]\left(\begin{array}{l}
p_{i}^{t} \\
V
\end{array}\right)
$$

In these equations $\left\{M_{p p}, \quad K_{p p}\right\} \in \mathfrak{R}^{M \times M}, \quad\left\{M_{c c}, \quad K_{c c}\right\} \in \mathfrak{R}^{N \times N}, \quad M_{c p} \in \mathfrak{R}^{N \times M}$, $K_{p c} \in \mathfrak{R}^{M \times N}, F_{p} \in \mathfrak{R}^{M \times 1}$, and $F_{V} \in \mathfrak{R}^{M \times k}$. The different quantities in the above equation are given by:

$$
\begin{aligned}
& M_{p p}^{U}=M_{p p}^{L}=\operatorname{diag}\left\lfloor\rho_{p} h_{p}\right\rfloor \\
& M_{c c}^{U}=M_{c c}^{L}=\operatorname{diag}\left[\frac{1}{c_{o}^{2}}\right] \\
& M_{c p}^{U}=\rho_{o}\left[\frac{(-1)^{i} A_{i}}{\sqrt{L_{z c}}} B x_{j i}^{(c)} B y_{j i}^{(c)}\right] \\
& M_{c p}^{L}=-\rho_{o}\left[\frac{A_{i}}{\sqrt{L_{z c}}} B x_{j i}^{(c)} B y_{j i}^{(c)}\right] \\
& K_{p p}^{U}=K_{p p}^{L}=2 D\left[I x_{j i} I y_{j i}\right\rfloor+D \operatorname{diag}\left\lfloor I x_{i}+I y_{i}\right\rfloor \\
& K_{c c}^{U}=K_{c c}^{L}=\operatorname{diag}\left[\frac{l_{j}^{2} \pi^{2}}{L_{x c}^{2}}+\frac{m_{j}^{2} \pi^{2}}{L_{z c}^{2}}+\frac{n_{j}^{2} \pi^{2}}{L_{z c}^{2}}\right] \\
& K_{p c}^{U}=\left[-\frac{(-1)^{j} A_{j}}{\sqrt{L_{z c}}} B x_{j i}^{(p)} B y_{j i}^{(p)}\right]
\end{aligned}
$$




$$
\begin{aligned}
& K_{p c}^{L}=\left[-\frac{A_{j}}{\sqrt{L_{z c}}} B x_{j i}^{(p)} B y_{j i}^{(p)}\right] \\
& F_{p}^{U}=\left(-\int_{A_{p}} \alpha_{i} \beta_{i} p_{U i}^{s} d A_{p}\right) \\
& F_{V}^{U}=\left[-\int_{A_{p}} \alpha_{i} \beta_{i} \frac{\left(h_{p}+h_{p z t}\right) E_{p z t} d_{31}}{(1-v)} \nabla^{2} \chi_{U}\left(x_{j}, y_{j}\right) \mathrm{d} A_{p}\right]
\end{aligned}
$$

The matrices $M_{c p}$ and $K_{p c}$ describe the structural-acoustic coupling, while the matrices $K_{p p}$ represent the panel stiffness matrix. Equations (20) represent the timedomain model developed for the system shown in Figure 1. After determining the modal amplitudes from these equations, the panel displacements $w(x, y ; t)$ and the pressure fields inside the cavity $p(x, y, z ; t)$ can be obtained from the following relations:

$$
\left\{\begin{array}{c}
w_{U}(x, y ; t) \\
p(x, y, z ; t) \\
w_{L}(x, y ; t)
\end{array}\right\}=\left[\begin{array}{ccc}
C_{U}^{(w)}(x, y) & 0 & 0 \\
0 & C^{(p)}(x, y, z) & 0 \\
0 & 0 & C_{L}^{(w)}(x, y)
\end{array}\right]\left\{\begin{array}{c}
\eta_{U}(t) \\
q(t) \\
\eta_{L}(t)
\end{array}\right\}
$$

where

$$
\begin{gathered}
C_{U}^{(w)}=\left[\alpha_{i}(x) \beta_{i}(y)\right] \\
C^{(p)}=\left[\psi_{i}(x) \varphi_{i}(y) \Gamma_{i}(z)\right] \\
C_{L}^{(w)}=\left[\alpha_{i}(x) \beta_{i}(y)\right]
\end{gathered}
$$

\section{NUMERICAL RESULTS}

Here, the numerical results obtained from the analytical model developed in this chapter are presented. The natural frequencies of the clamped panel have been calculated by using the following approximate formula, which is based on an energy (Raleigh) technique [5]:

$$
f_{i j}=\frac{\pi}{2} \sqrt{\frac{D}{\rho_{p} h_{p}}\left[\frac{G_{i}^{4}}{L_{x p}^{4}}+\frac{G_{j}^{4}}{L_{y p}^{4}}+\frac{2 H_{i} H_{j}}{L_{x p}^{2} L_{y p}^{2}}\right]}
$$

where the dimensionless parameters $\mathrm{G}$ and $\mathrm{H}$ are given in [5]. These parameters are functions of the indices $i$ (in the $x$ direction) and $j$ (in the $y$ direction) and the boundary conditions for the plate. The cavity natural frequencies are calculated through the following equation [1]: 


$$
f_{i j k}=\frac{c_{o}}{2 \pi} \sqrt{\left(\frac{l_{i} \pi}{L_{x c}}\right)^{2}+\left(\frac{m_{i} \pi}{L_{y c}}\right)^{2}+\left(\frac{n_{i} \pi}{L_{z c}}\right)^{2}}
$$

$$
; i=0,1,2, \ldots . \quad j=0,1,2, \ldots . \quad k=0,1,2, \ldots .
$$

where the indices $l_{i}, m_{j}$, and $n_{k}$ are associated with the spatial functions of the $i^{\text {th }}$ rigid cavity mode in the $x, y$, and $z$ directions respectively. The first few natural frequencies of the uncoupled and coupled system are tabulated as shown in Table 1.

\begin{tabular}{|c|c|c|c|c|c|c|c|}
\hline \multicolumn{7}{|c|}{ Uncoupled System } & \multirow{3}{*}{$\begin{array}{c}\text { Coupled system } \\
\begin{array}{c}\text { Panels-Cavity-Panel } \\
\text { System }\end{array}\end{array}$} \\
\hline & \multicolumn{3}{|c|}{ Cavity } & \multicolumn{3}{|c|}{ Panel } & \\
\hline \multicolumn{3}{|c|}{ Mode } & \multirow{2}{*}{$\begin{array}{c}\text { Closed Form } \\
\text { Formula } \\
281.33\end{array}$} & \multicolumn{2}{|c|}{ Mode } & \multirow{2}{*}{$\begin{array}{c}\text { Blevins's } \\
\text { Approximate Formula } \\
44.452\end{array}$} & \\
\hline 1 & 0 & 0 & & 1 & 1 & & 42.75 \\
\hline 0 & 0 & 1 & 337.6 & 2 & 1 & 76.017 & 43.85 \\
\hline 0 & 1 & 0 & 375.11 & 1 & 2 & 103.61 & 67.447 \\
\hline 1 & 0 & 1 & 439.45 & 3 & 1 & 127.66 & 68.407 \\
\hline 1 & 1 & 0 & 468.89 & 2 & 2 & 132.78 & 132.98 \\
\hline 0 & 1 & 1 & 504.66 & 3 & 2 & 181.7 & 134.11 \\
\hline & & & & & & & 180.24 \\
\hline & & & & & & & 181.31 \\
\hline & & & & & & & 285.41 \\
\hline
\end{tabular}

Table 1. The undamped natural frequencies $(\mathrm{Hz})$ of the uncoupled and coupled system

Due to the complexity of the structural-acoustic coupling of this system, the effect of the stiffness coupling matrix $K_{p c}$ and the inertia coupling matrix $M_{c p}$ on the coupled natural frequencies cannot be easily realized. In fact, the entries of $M_{c p}$ increase the values of the first few acoustic resonance frequencies above their uncoupled values, hence, contributing a "mass reduction" effect. On the other hand, the entries of $K_{p c}$ decrease the values of the low (vibration) resonance frequencies below their uncoupled values, hence, contributing a "stiffness reduction" effect.

\section{CONTROL APPROACH}

\subsection{State space design method}

The idea of state space comes from the state-variable method of describing differential equations [6]. The differential equations for a dynamic system can be represented in the state-variable-form vector equation

$$
\dot{x}=A x+B u
$$

where the input is $u$ and the output is

$$
y=C x+D u
$$

The column vector $x$ is called the state of the system and contains $n$ elements in an $\mathrm{n}^{\text {th }}$-order system. For mechanical systems, the state vector elements consist of the positions and velocities of the separate bodies. The quantity $A$ is an $n \times n$ system 
matrix; $\mathrm{B}$ is an $n \times 1$ input matrix, $\mathrm{C}$ is a $1 \times n$ row matrix referred to as the output matrix, and $D$ is a scalar called the direct transmission term.

\subsection{Multiple input-multiple output System}

In case of disturbance input, a multiple input multiple output system (MIMO) is suggested. Eq. (26) can be rewritten by using transformation matrices $T_{d}$ and $T_{u}$ as follows

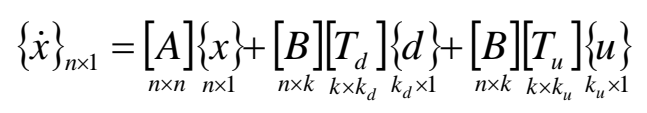

where

$$
\left[T_{d}\right]_{k \times k_{d}}=\left[\begin{array}{c}
{\left[I_{k_{d} \times k_{d}}\right]} \\
{[0]}
\end{array}\right], \quad\left[T_{u}\right]_{k \times k_{u}}=\left[\begin{array}{c}
{[0]} \\
{\left[I_{k_{u} \times k_{u}}\right]}
\end{array}\right], \quad\{U\}_{k \times 1}=\left\{\begin{array}{l}
\{d\}_{k_{d} \times 1} \\
\{u\}_{k_{u} \times 1}
\end{array}\right\}
$$

\subsection{Optimal control design}

An optimal control system seeks to maximize the return from a system at the minimum cost. In general terms, the optimal control problem is to find a control $u$ which causes the system $\dot{x}=g(x(t), u(t), t)$ to follow an optimal trajectory $x(t)$ that minimizes a performance criterion, or cost function $J=\int h(x(t), u(t), t) d t$. The most effective and widely used method to design a full state feedback control for linear systems is the optimal Linear Quadratic Regulator (LQR). The control law that minimizes $J$ is given by the linear state feedback

$$
u=-k x
$$

A more general form of the performance function is

$$
J=\int_{0}^{\infty}\left[x^{T} Q x+u^{T} R u\right] d t
$$

where $Q$ is a symmetric positive semi-definite state weighting matrix, and $R$ is a symmetric positive definite control effort weighting. Eq. (31) reduces to a simpler form [6] if we take $Q=\rho C^{T} C$ and $R=I$.

$$
J=\int_{0}^{\infty}\left[\rho y^{2}(t)+u^{2}(t)\right] d t
$$

\subsection{Estimator design}

Since measuring the states is not possible, so estimation is the choice. If the estimate of the states is denoted by $\hat{x}$, then it will be convenient to replace the true state in the control law given by Eq. (30) by the estimates. The control becomes

$$
u=-K \hat{x}
$$


One method of estimating the state is to construct a full-order model of the plant dynamics,

$$
\dot{\hat{x}}=A \hat{x}+B u
$$

This estimator will be satisfactory if we can obtain the correct initial condition $x(0)$ and set $\hat{x}(0)$ equal to it. The equation for this scheme will be:

$$
\dot{\hat{x}}=A \hat{x}+B u+L(y-C \hat{x})
$$

Here $L$ is a proportional gain defined as

$$
L=\left[\begin{array}{llll}
l_{1} & l_{2} & \cdots & l_{n}
\end{array}\right]
$$

and is chosen to achieve satisfactory error characteristics. The dynamics of the error can be obtained as

$$
\dot{e}=(A-L C) e
$$

\subsection{Combined control law and estimator}

If we take the control-law design described in Section 4.3, combined with the estimator design described in Section 4.4, the overall system dynamics in the state form is (Figures 2)

$$
\left[\begin{array}{c}
\dot{x} \\
\dot{e}
\end{array}\right]=\left[\begin{array}{cc}
A-B K & B K \\
0 & A-L C
\end{array}\right]\left[\begin{array}{l}
x \\
e
\end{array}\right]
$$

The response of the system depends mainly on the input signals that excite it. For plate-cavity-plate system, the upper plate is excited by the disturbance pressure which may take several shapes. The external disturbance applied to the top of the panel is expected to take one of the following types: (i) Impulse pressure: a sudden change of the ambient pressure above the panel, (ii) Damped periodic pressure: will be described by a damped sine wave, and (iii) Random pressure: a varying pressure wave in frequency and amplitude in a random fashion.

\section{SIMULATION RESULTS}

Using the previous analysis, numerical simulation is applied to the system. The objective in the simulation is to reduce the pressure inside the cavity. Figs. 3 to 5 illustrate the resulting pressure response for different input types. It is observed that the maximum amplitude and the settling time are highly reduced and the response can be tuned to a certain values by adjusting the weighting factor used in the control model. 


\section{CONCLUSIONS}

In this work, model based on time domain state space approach have been developed for an active structural-acoustic control (ASAC) application. The resulting control schemes have been studied for reduction of the noise transmitted through a plate-cavity-plate system. It has been demonstrated that "appropriate" choices of the controller design parameter can result in decreasing the settling time required to damp the noise inside the cavity. Comprehensive mechanics-based analytical models have been developed to predict the structural-acoustic interactions in the case of Plate-cavity-plate system, where one plate is placed in the far field of a noise source at the top of the cavity, and the other is placed at the bottom of the cavity. Piezoelectric patches, which are bonded symmetrically to the top and bottom surfaces of the top plate, are used as actuators, and the acoustic pressure is calculated inside the cavity. The developed models have the following advantages: (i) They are capable of predicting the structural-acoustic interactions, and (ii) They take into account the coupling between the plate vibration and the pressure inside the cavity. The control scheme that has been developed throughout this work is combined optimal control law and estimator.

The numerical results obtained for the coupled system show how the vibration and acoustic fields interact with each other. It was found that the entries of the mass matrix $M_{c p}$ increase the values of the first few acoustic resonance frequencies above their uncoupled values. On the other hand, the entries of the stiffness matrix $K_{p c}$ decrease the values of the low vibration resonance frequencies below their uncoupled values. Using the combined optimal control law and estimator scheme it is observed that this technique is efficient in reducing the maximum amplitude, and the settling time.

\section{REFERENCES}

[1] Kinsler L. E., Frey A. R., Coppens A. B., and Sanders J. V., Fundamentals of Acoustics, Fourth Edition, John Wiley and Sons, Inc., New York, (2000).

[2] Kuttruff H., Room Acoustics, 2nd Edition, Spon Press, New York, 1981.

[3] Balachandran B., Sampath A. and Park J, "Active control of interior noise in a three-dimensional enclosure", Smart Materials and Structures 5, 89-97 (1996).

[4] Moustafa Al-Bassyiouni and Balachandran B., "Sound transmission through a flexible panel into an enclosure: structural-acoustic model", Journal of Sound and Vibration 284, 467-486 (2005).

[5] Blevins J., Formulas for Natural Frequency and Mode Shapes, Van Nostrand Reinhold Company, (1978).

[6] Franklin G. N., Powell J. D., and Emami A. N., Feedback control of dynamic systems, Pearson Prentic Hall, (2006). 


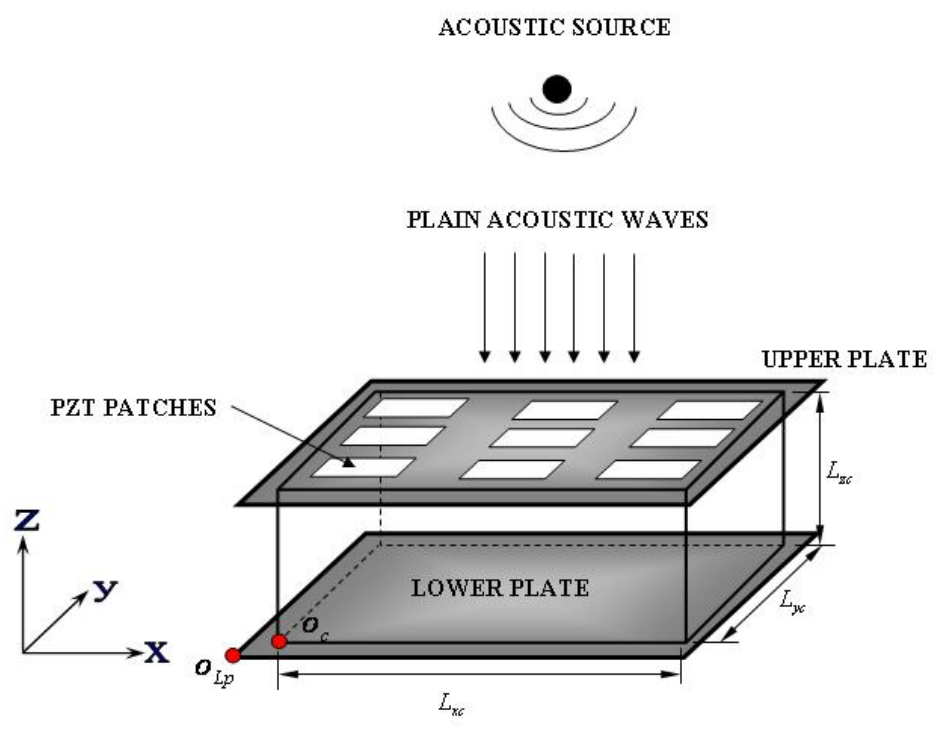

(a)

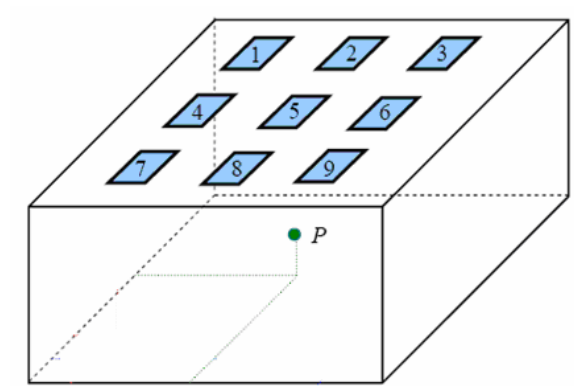

(b)

Fig 1. (a) Schematic of the panel-cavity-panel system used for the analysis model, (b) Centres locations of the actuator pairs on the plate and locations of the calculated pressure in the cavity

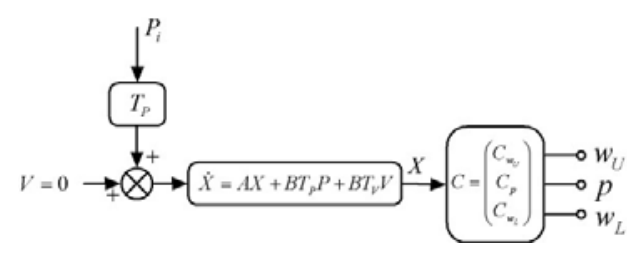

(a)

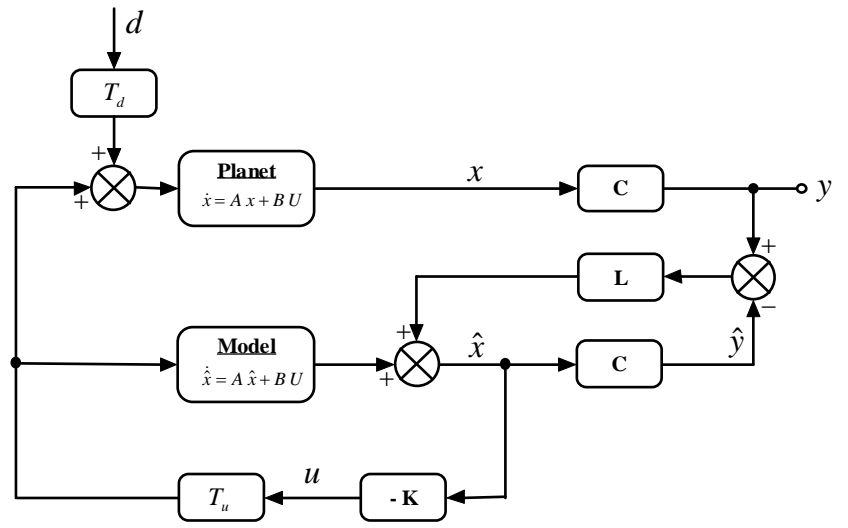

(b)

Fig 2. (a) Open loop block diagram for the panel-cavity-panel system, (b) Assumed closed loop system for the combined optimal control-law and estimator design in case of a disturbance input $d$ 
Impulse Input Response

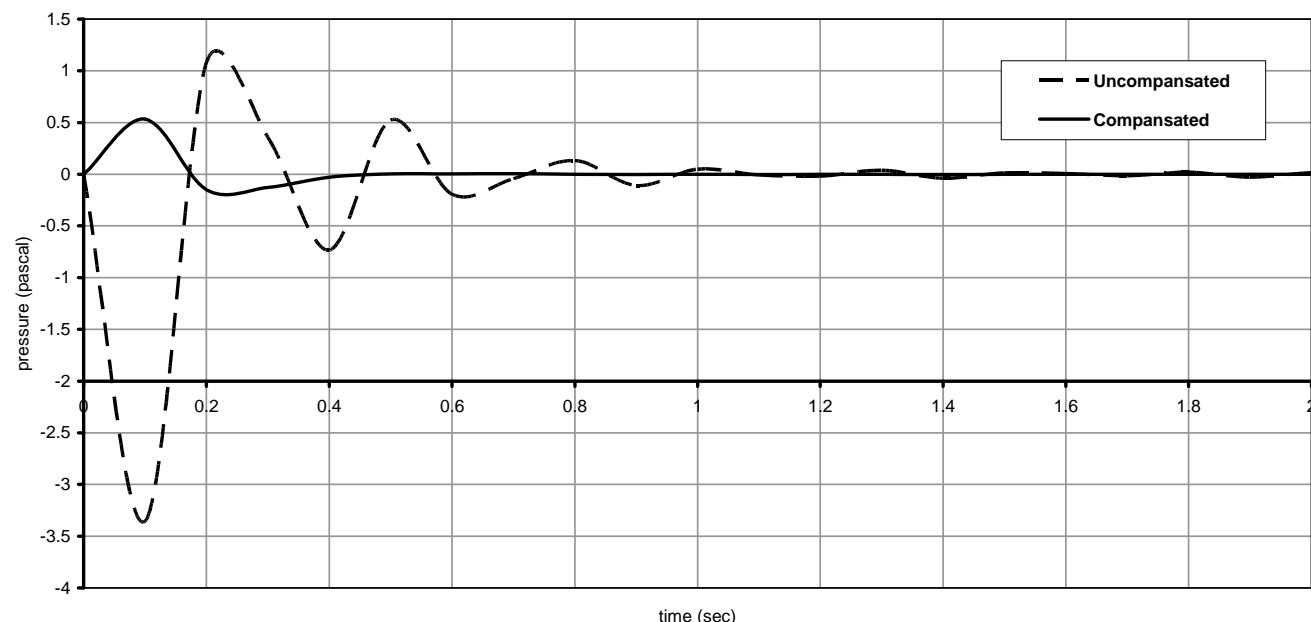

Fig 3. Uncompensated and compensated time responses due to impulse pressure input

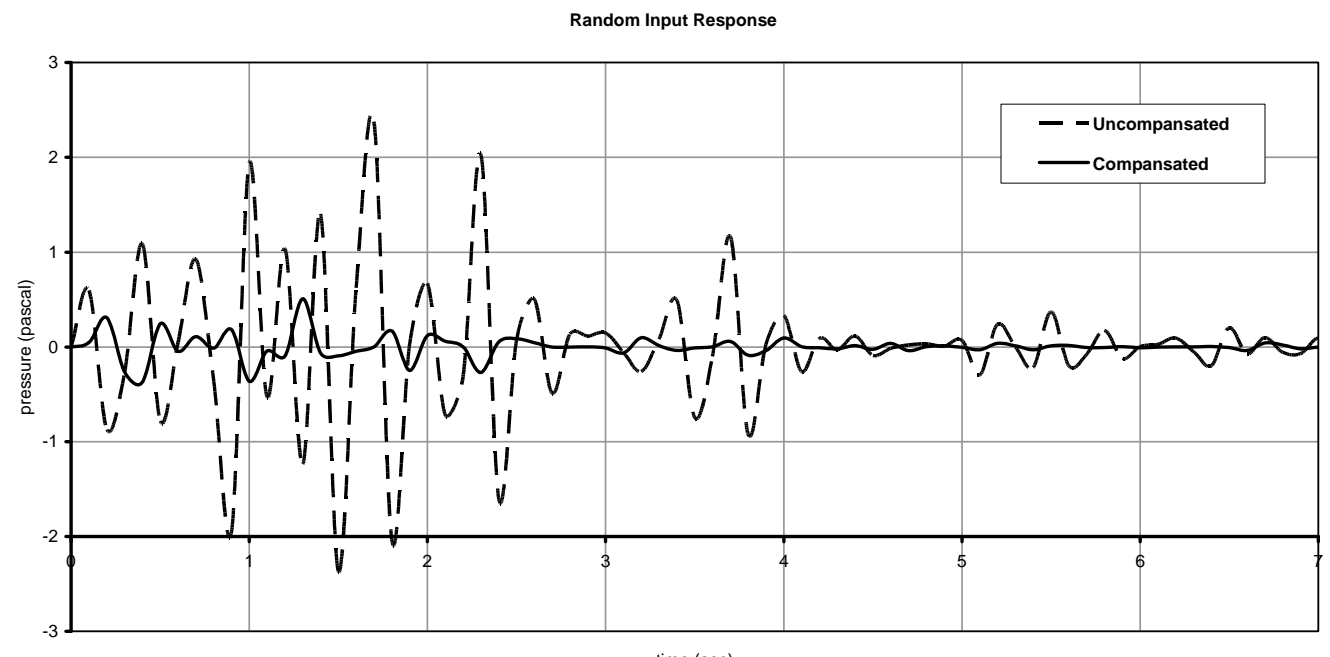

Fig 4. Uncompensated and compensated time responses due to random pressure input

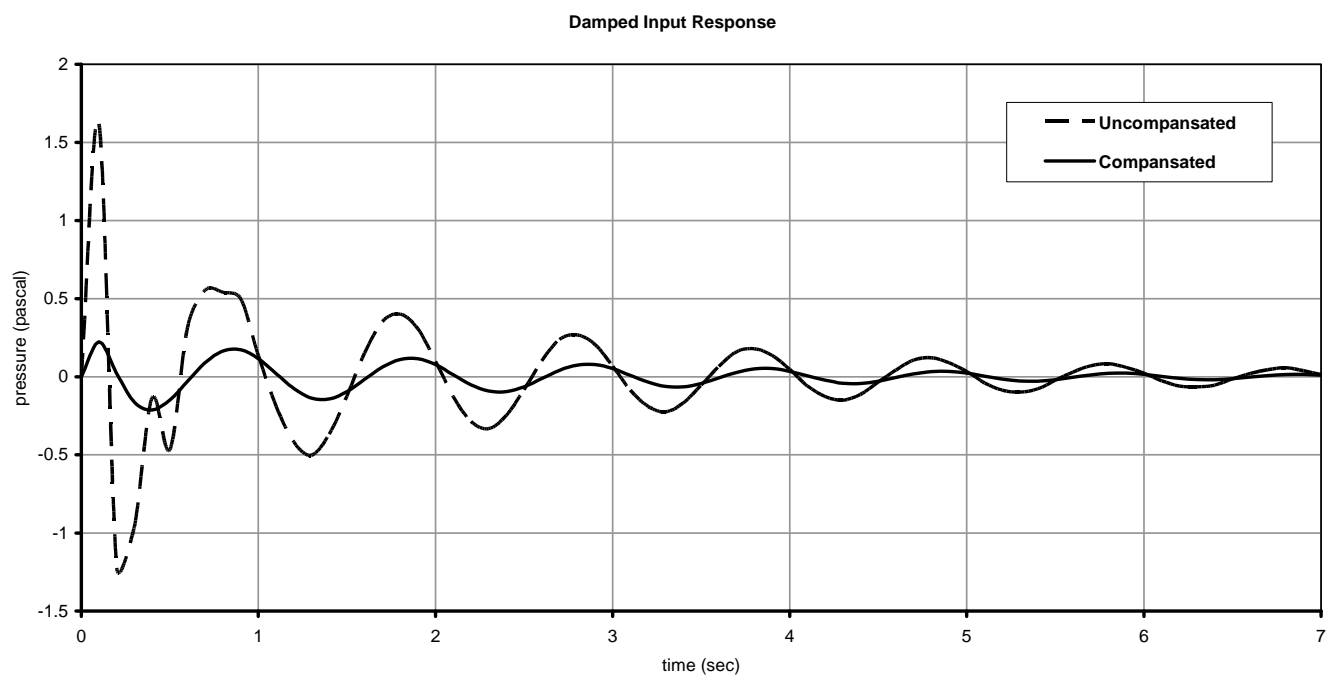

Fig 5. Uncompensated and compensated time responses due to damped pressure input 